\title{
Estudo dos critérios de falhas em flexão em Compósitos PPS/Fibras de Carbono utilizando elementos finitos
}

\author{
Failure criterious study in flexure of PPS/Carbon biber composites by using \\ finite elements
}

Artigo

Original

Eder José Siqueira ${ }^{1}$

Maria Cândida Magalhães de Faria ${ }^{2}$

Fernando de Azevedo Silva ${ }^{3}$

Edson Cocchieri Botelho ${ }^{3}$

Original

Paper

\section{Palavras-chaves: \\ Compósito \\ Termoplástico PPS/C}

Elementos Finitos

Critérios de Falha

\section{Resumo}

O constante desenvolvimento dos materiais aliado à necessidade de se obter componentes estruturais de alto desempenho como materiais alternativos na indústria aeronáutica, bem como, considerando a aplicabilidade atual dos compósitos estruturais, previsões de falha da estrutura e análise das cargas máximas que as mesmas podem suportar se tornam tópicos fundamentais de pesquisas com a finalidade de garantia da confiabilidade quanto ao emprego dos mesmos em diversas áreas. Considerando que o compósito termoplástico PPS/C - Poli (sulfeto de fenileno) reforçado com fibras de carbono - está surgindo como uma alternativa para a substituição de compósitos poliméricos termorrígidos para aplicações específicas e que ainda há escassez na literatura atual de dados relativos à previsão de falhas, este trabalho tem como objetivo realizar uma analise bidimensional do comportamento mecânico do compósito PPS/C sob carregamento estático linear em flexão de três pontos. Neste estudo, foram analisados os critérios de falha de máxima tensão, critérios polinomiais dos índices de força e das relações inversas dos índices de força de Tsai-Wu, de Hill e de Hoffman. Por fim, foram comparados os resultados obtidos a partir do modelo computacional com resultados experimentais obtidos a partir de ensaios de flexão com o objetivo de analisar, avaliar e validar a simulação numérica utilizada.

\begin{abstract}
The continuous development of materials associated with the need to obtain structural components of high performance that can serve the purpose of alternate material for the aircraft industry; in addition to the current applicability of the structural composite, structural failure predictions, as well as the maximum loads that such structures may have the capacity to bear, have become the subject-matter to a number of fundamental researches, which purpose is to guarantee the reliability of such materials, so that they can be duly applied in a variety of fields. Taking into account that the thermoplastic composite of PPS/C - Poly(phenylene-sulfide) reinforced with carbon fibers, is springing up as an alternative to the thermoset polymers composites, besides the fact that the current literature lacks data regarding failure predictions, the present paper limits itself to the
\end{abstract}

\footnotetext{
Mestre e Docente - Centro Universitário de Volta Redonda - UniFOA / Universidade Estadual Paulista - Unesp - FE - Campus Guaratinguetá/SP

${ }^{2}$ Mestre - Unesp - Universidade Estadual Paulista - FE - Campus Guaratinguetá/SP

${ }^{3}$ Doutor e Docente - Unesp - Universidade Estadual Paulista - FE - Campus Guaratinguetá/SP
} 
two-dimensional analysis of PPS/C composite type mechanical behavior under a linear static loading at a three point bending. In this study, the maximum stress criteria, the strength ratio, and the reversed relation of strength ratio of Tsai-Wu, as well as the criteria of Hill and Hoffman to predict failure, were analyzed. At last, a comparison was made between the results obtained from the computer model against the experimental results. The purpose of such comparison was to analyze, assess and validate the numerical simulation as a supplement to the experimental trials.

\section{Introdução}

O uso de compósitos em estruturas, principalmente os reforçados com fibras de carbono, tem permitido uma significativa redução no peso destas, bem como contribuído para melhorar as resistências à corrosão e à fadiga de uma infinidade de componentes de aeronaves de última geração, plataformas marítimas de petróleo, satélites, submarinos, foguetes, veículos automotores, trens de alta velocidade e de implantes ortopédicos, dentre outras aplicações [1-3].

Segundo TITA et al [4] pode-se definir que a falha do componente ocorre no momento em que o mesmo não pode mais satisfazer a função para o qual foi projetado. Sendo, assim, um critério de falha visa fornecer uma interpretação dos efeitos provocados por carregamento combinados (estado duplo de tensões) na estrutura, indicando quando houve uma eventual falha local ou global por parte da mesma. Dentro deste contexto torna-se importante a avaliação de critérios de falhas objetivando o estudo do comportamento mecânico de laminados estruturais de uma forma mais rápida sem que exista a necessidade da utilização de uma grande quantidade de material a ser submetido a uma série de ensaios mecânicos tais como tração, compressão, flexão, etc. Para esta finalidade, o aplicativo computacional ANSYS vem sendo utilizado com sucesso permitindo a realização de simulações numéricas de vários critérios de falhas podendo ser citados os critérios: Hill, Hoffman, Tsai-Wu, tensão máxima, dentre outros [4-7].

O critério de tensão máxima afirma que as tensões aplicadas nas direções principais do material devem ser menores que as resistências nas respectivas direções de carga, ou seja, apresenta assim três modos possíveis de falhas, portanto:

$$
\begin{aligned}
& \text { Falhas em Tração: }\left\{\begin{array}{l}
\sigma_{1}<X_{t} \Rightarrow \text { Modo de falha } 1 ; \\
\sigma_{2}<Y_{t} \Rightarrow \text { Modo de falha } 2 ;
\end{array}\right. \\
& \text { Falhas em Compressão: }\left\{\begin{array}{l}
\sigma_{1}>-X_{c} \Rightarrow \text { Modo de falha } 1 \\
\sigma_{2}>-Y_{c} \Rightarrow \text { Modo de falha } 2
\end{array}\right.
\end{aligned}
$$

Os modos de falhas indicados não são precisos, apenas prováveis. Supõe-se que o material falha pelo mecanismo associado ao valor de $X_{t}, X_{c}, Y_{t}, Y_{c}$ e $S$ conforme a inequação que foi violada primeiramente na falha [8].
O critério polinomial de Hill diz que para o estado plano de tensões (EPT), que é relativo a este estudo, pode-se considerar $\sigma_{3}=\tau_{23}=\tau_{13}=0$, sendo que a equação, em que Hill considera a seguinte formulação:

$$
(G+H) \sigma_{1}^{2}+(F+H) \sigma_{2}^{2}-2 H \sigma_{1} \sigma_{2}+2 N \tau_{12}^{2}=1
$$

Já o critério polinomial de Tsai-Wu afirma que de forma geral, para o EPT, a teoria pode ser apresentada da seguinte forma, quando considerado $\sigma_{3}=\tau_{23}=\tau_{13}=0$ :

$$
\mathrm{F}_{1} \sigma_{1}+\mathrm{F}_{2} \sigma_{2}+\mathrm{F}_{6} \tau_{12}+\mathrm{F}_{11} \sigma_{1}^{2}+\mathrm{F}_{22} \sigma_{2}^{2}+\mathrm{F}_{66} \tau_{12}{ }^{2}+2 \mathrm{~F}_{12} \sigma_{1} \sigma_{2}+\mathrm{F}_{16} \sigma_{1} \tau_{12}+\mathrm{F}_{26} \sigma_{2} \tau_{12}=1
$$


O critério de Hoffman pode ser visto como uma extensão do critério de Hill ou uma simplificação do critério de Tsai-Wu. Em relação ao critério de Hill, é feita a inclusão de termos lineares de diferenças entre resistências de tração e compressão. Assim, o critério de Hoffman tem sido bastante utilizado tanto em problemas de plasticidade de metais quanto em falha frágil de laminados fibrosos [9-10]. Este trabalho tem como objetivo realizar uma análise bidimensional do comportamento mecânico do compósito PPS/C sob carregamento estático linear em flexão de três pontos, sendo analisados os critérios de falha de máxima tensão, polinomiais dos índices de força e das relações inversas dos índices de força de Tsai-Wu, de Hill e de Hoffman. Os resultados obtidos a partir da simulação por ANSIS foram também comparados com os obtidos experimentalmente.

\section{Experimental}

\subsection{Materiais}

Neste trabalho foi estudado um compósito estrutural polimérico com matriz termoplástica PPS -Poli (Sulfeto de Fenileno) reforçado com tecidos de fibras de carbono (configuração 5HS "satin weave"), fornecido pela empresa holandesa Ten Cate Advanced Composites. O conteúdo volumétrico de fibras foi de $61 \%$ avaliado pelo método de digestão ácida em trabalhos anteriores [8]. Os laminados estudados apresentaram configuração $\left[0^{\circ} / 90^{\circ}\right]_{3 \mathrm{~s}}$, e foram devidamente analisados por ensaios de ultrassom e microscopia óptica, com a principal finalidade de garantir a qualidade das mesmas na realização dos ensaios experimentais.

\subsection{Simulação em flexão de três pontos}

Para o desenvolvimento deste trabalho, ensaios de cisalhamento Iosipescu e flexão em três pontos foram realizados com base nas normas ASTM-D5379 e DIN-EN-2562, respectivamente, em uma máquina de ensaios universal Shimadzu, modelo SFL-50kN.

A simulação numérica foi pré-processada, processada e pós-processada pelo aplicativo computacional ANSYS, sendo que os dados experimentais foram tomados como referência para avaliar as tensões de resistência do material PPS/C em flexão na simulação numérica, com o principal objetivo de validar a sua utilização na análise de falha de materiais compósitos.

Inicialmente, no ANSYS, para se realizar uma análise de falha por flexão de três pontos utilizando o método dos elementos finitos, devese, por meio de um pré-processador, criar pontos chaves, que definem as dimensões geométricas, ou seja, delimitam o corpo em análise. Estes pontos chaves são unidos por linhas para se obter por fim um elemento geométrico de área nas dimensões do corpo de prova, ou seja, um elemento de área retangular de $55 \mathrm{~mm}$ x $10 \mathrm{~mm}$, pois essa análise se relaciona a um laminado bidimensional em que o comprimento e a largura são excessivamente maiores que a espessura. Introduz-se a partir daí as características mecânicas do laminado em análise, sua discretização em elementos finitos, a restrição ao movimento e condições de carregamento, para se obter a solução do problema, conforme apresentado na Figura 1.

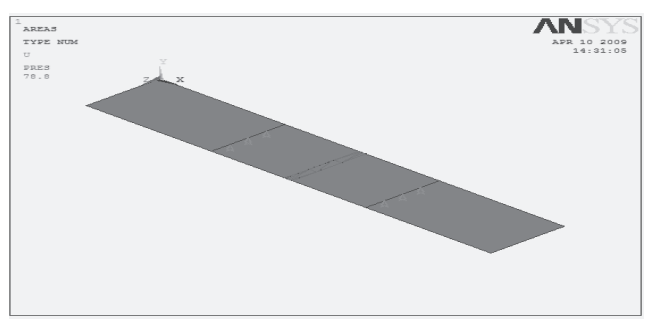

Figura 1 - Modelo do laminado para simulação em flexão de três pontos.

Neste trabalho, os valores encontrados a partir dos modelos utilizados foram comparados com os valores obtidos experimentalmente.

\section{Resultados e Discussão}

\subsection{Resultados experimentais}

Os resultados experimentais de resistências à flexão e ao cisalhamento Iosipescu, encontram-se apresentados na Tabela 1. Em ambos os casos, as tensões encontradas para estes laminados termoplásticos quando comparadas a valores de outros compósitos tradicionalmente utilizados pela indústria aeroespacial tais como os laminados de resina epóxi/fibras de carbono, mostram-se superiores, podendo em alguns casos ser observada uma melhora de até 30\% [9]. Como dito anteriormente, os ensaios experimentais foram realizados com o único propósito de validar os resultados obtidos a partir da simulação por Ansys. 
Tabela 1 - Resultados experimentais de flexão e cisalhamento Iosipescu para os laminados de PPS/C.

\begin{tabular}{|c|c|c|c|}
\hline $\begin{array}{c}\text { Resistência à } \\
\text { flexão } \text { (MPa) }\end{array}$ & $\begin{array}{c}\text { Módulo em flexão } \\
\text { (GPa) }\end{array}$ & $\begin{array}{c}\text { Resistência ao cisalhamento } \\
\text { Iosipescu (MPa) }\end{array}$ & $\begin{array}{c}\text { Módulo de cisalhamento } \\
\text { Iosipescu (GPa) }\end{array}$ \\
\hline $1340 \pm 29$ & $60,7 \pm 1,1$ & $109,2 \pm 0,4$ & $4,9 \pm 0,7$ \\
\hline
\end{tabular}

\subsection{Resultados da simulação numérica}

Com base no dimensionamento dos corpos-de-prova e valores médios dos carregamentos, obtidos a partir dos ensaios experimentais em flexão de três pontos e cisalhamento Iosipescu, realizaram-se as simulações numéricas no aplicativo computacional ANSYS. Considerando as informações fornecidas pelo aplicativo computacional acoplado ao equipamento SHIMADZU SFL-50kN, obteve-se os valores das cargas e dos deslocamentos do laminado compósito em flexão de três pontos, entretanto, estes valores foram transferidos para o aplicativo computacional EXCEL e foram comparados entre si, de onde foi extraído o carregamento médio de 797,03N, que é transformado numa tensão de 79,70 MPa para alimentar a simulação. A simulação numérica indica que o laminado rompe a uma tensão de $788 \mathrm{MPa}$, equivalente a aplicação de um carregamento numa área de $10 \mathrm{~mm}^{2}$ de $78,8 \mathrm{~N}$, conforme representado na Figura 1.

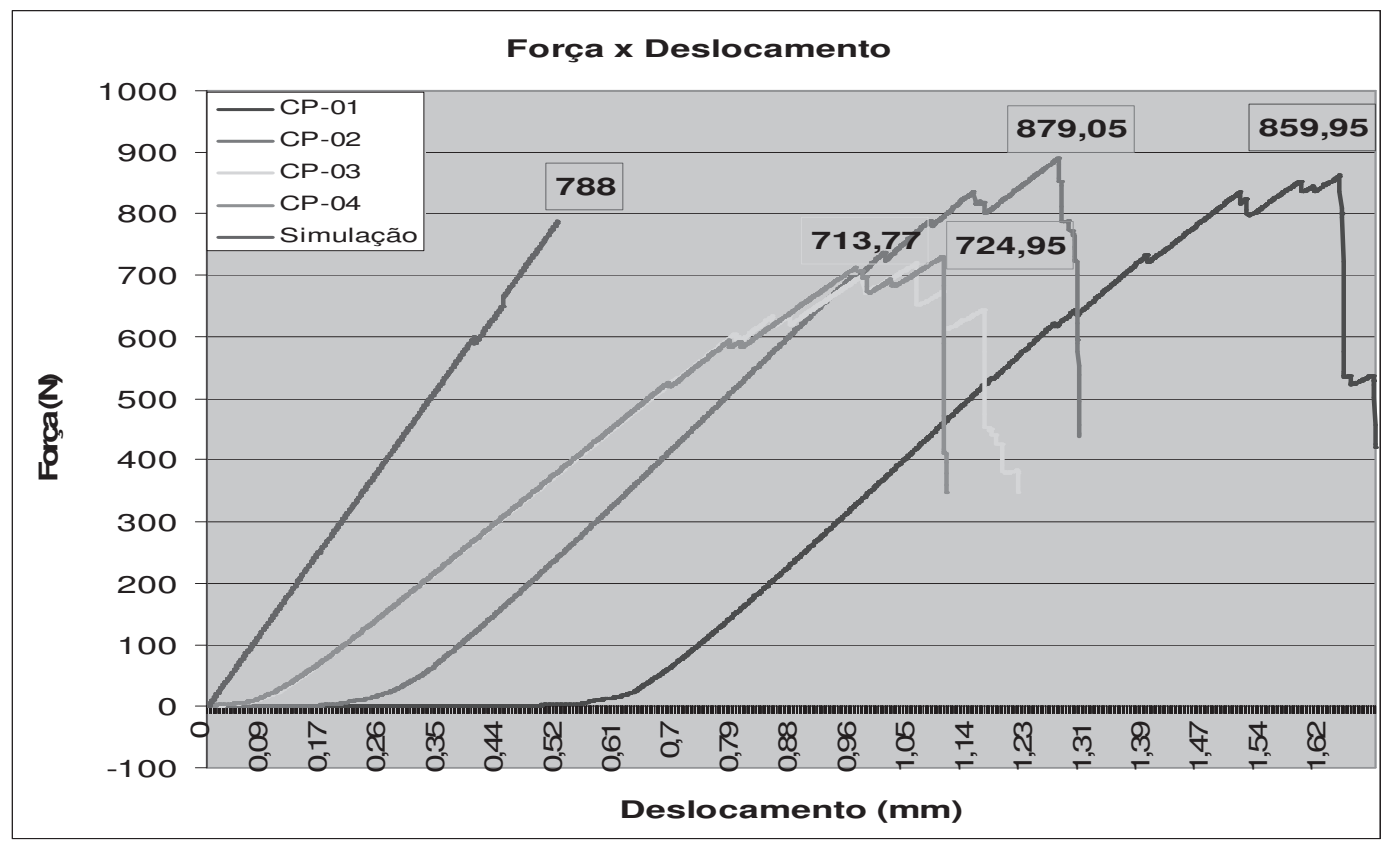

Tabela 1 - Resultados experimentais de flexão e cisalhamento Iosipescu para os laminados de PPS/C.

A partir deste gráfico pode ser observado que existe uma diferença significativa entre o comportamento do laminado PPS/C submetido a ensaio de flexão em três pontos, ou seja, os valores apresentados demonstram que o material antes de romper apresenta alterações no comportamento elástico linear. Este comportamento se dá em função do descolamento das fibras em relação à matriz e também em relação ao efeito das tensões cisalhantes do plano do laminado. Ainda a partir destes ensaios foi observado que a ruptura do laminado em flexão de três pontos se dá na ultima lâmina onde as fibras estão dispostas a $90^{\circ}$.
Apesar das diferenças dos comportamentos viscoelásticos, os valores experimentais de resistência à flexão são próximos aos valores obtidos por simulação numérica. Desta forma, os valores encontrados são bastante confiáveis e a simulação em si pode ser utilizada em complementação/substituição em alguns casos específicos, quando se tem pré-definidas as propriedades do compósito, suas condições geométricas e de carregamento, aos ensaios que são mais dispendiosos em termos de mão de obra e tempo de execução. Um projetista pode a partir do conhecimento do processo pelo qual o material esteja sendo solicitado e 
da forma como reage a esta solicitação, assim como a partir do conhecimento do processo de simulação num programa computacional, estimar com boa aproximação as tensões apropriadas de trabalho do material compósito.

Concluiu-se então que além de ser um dos mais aplicados em análise de falha inicial em materiais compósitos, os critérios polinomiais de índices de forças ou das relações inversas dos índices de forças de Tsai-Wu são perfeitamente adequados para se trabalhar com segurança em termos da previsão inicial de falha de um compósito termoplástico sob regime estático de carregamento. A partir dos dados obtidos neste trabalho também foi observado que para previsão de falhas em compósitos PPS/C, o critério de falha de máxima tensão também se mostrou adequado, podendo assim, ser utilizado para análise de previsão inicial de falhas em compósitos termoplásticos do tipo PPS/C.

Para efeito comparativo foram implementados neste trabalho por meio do aplicativo EXCEL, dois critérios polinomiais de previsão inicial de falha, sendo estes: $\mathrm{O}$ critério de Hill e o critério de Hoffman.

Com relação ao critério de previsão inicial de falhas de Hill (considerado a base dos critérios polinomiais de Tsai-Wu e de Hoffman), observa-se que para a simulação em flexão de três pontos não foi observada falha do material com a tensão de ruptura apresentada pelo programa de cálculo. Este fato indica que se pode trabalhar com um acréscimo de carregamento. Entretanto, como o índice de falha de Hill ficou um pouco abaixo, mas bem próximo do limiar da falha (considerado neste caso como sendo 1), não se pode descartar ou dizer que o emprego deste critério foi inapropriado, pois para tanto deve-se promover a análise sob óticas diferenciadas de simulação, ou seja, o corpos-de-prova deveriam ser discretizados em elementos diferenciados, podendo assim reagir de forma que indicasse falha. Para este trabalho foi adotado uma malha de elemento finito quadrado de $1 \mathrm{~mm}^{2}$ e com esta foram realizados os cálculos, entretanto, não foi analisado com elemento finito em outra dimensão ou em outra característica geométrica. A Figura 2 e a Tabela 2 apresenta um estudo comparativo entre os índices de falhas obtidos no ANSYS e os implementados no EXCEL.

Já o critério polinomial de previsão inicial de falha de Hoffman, indica que para ambos os casos analisados o material falha, pois os índices de falha estão acima do limiar permitido e são maiores que os índices apresentados pelos critérios de máxima tensão, índices de força de Tsai-Wu e relação inversa dos índices de força de Tsai-Wu, contidos no ANSYS. Desta forma, se o projetista estiver trabalhando na análise de previsão inicial de falha de um laminado tipo PPS/C com este critério, estará indicando a aplicação de um carregamento abaixo da ruptura real do laminado, porém o critério de Hoffman não pode ser descartado para análise de compósitos termoplásticos, pois em ordem de grandeza o índice encontrado não se apresenta distante dos índices dos critérios analisados no ANSYS. Para se concluir sobre a exclusão de um critério, deve-se analisar sob condições diferenciadas de discretização e de carregamento.

A seguir é apresentado um estudo comparativo entre todos os critérios de falhas analisados neste trabalho, considerando os esforços em flexão de três pontos com uma tensão de $78,8 \mathrm{~N} / \mathrm{mm}^{2}$ (equivalente a uma força de ruptura de $788 \mathrm{~N}$ ), índices de falha conforme apresentados na Tabela 2 e na Figura 2.

Tabela 2 - Índices de falha em compósito PPS/C sob carregamento em flexão de três pontos.

\begin{tabular}{|c|c|c|c|c|c|}
\hline $\begin{array}{c}\text { CRITÉRIOS } \\
\text { DE FALHAS }\end{array}$ & $\begin{array}{c}\text { TENSÃO } \\
\text { MÁXIMA }\end{array}$ & HILL & HOFFMAN & TSAI-WU & $\begin{array}{c}\text { INVERSO } \\
\text { DE TSAIWU }\end{array}$ \\
\hline ÍNDICES & 1,004 & 0,989 & 1,025 & 1,002 & 1,001 \\
\hline
\end{tabular}




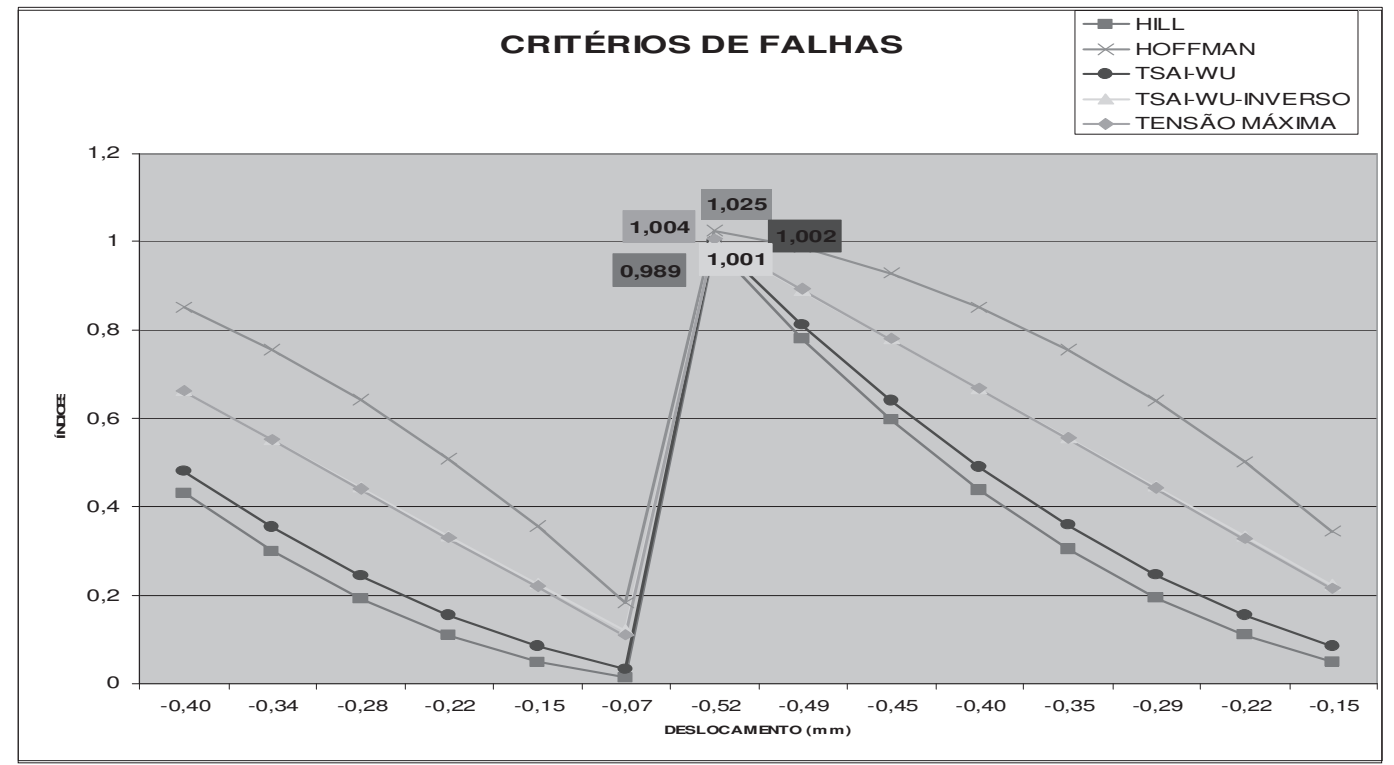

Figura 2 - Comparativos entre os critérios de falhas em flexão de três pontos. Análise para uma quantidade mais reduzida de nós.

Pode-se observar a partir dos dados apresentados na Tabela 2 e Figura 2 que os critérios de máxima tensão, de índice de forças de Tsai-Wu e das relações inversas dos índices de força de Tsai-Wu falharam com o carregamento bem próximo do carregamento real, entretanto, para este mesmo carregamento os critérios de Hill e Hoffman implementados no EXCEL com base nas tensões de tração e compressão nodais obtidas por meio do ANSYS se apresentaram um pouco abaixo e um pouco acima, respectivamente, do limiar da falha, indicando neste caso que para este carregamento e nas condições da simulação desenvolvida neste trabalho o critério de Hill não indica falha e o critério de Hoffman indica que pode trabalhar com um carregamento abaixo do carregamento calculado e indicado no ANSYS, ou seja, para as condições simuladas neste trabalho os índices de previsão de falhas de Hill e de Hoffman se mostraram menos e mais conservadores, respectivamente, para previsão inicial de falhas em compósitos termoplásticos do tipo PPS/C.

\section{Conclusões}

A partir deste trabalho, concluiu-se que o emprego da simulação numérica no ANSYS é válido, pois apresentou resultados numéricos aproximados dos resultados experimentais. Desta forma, o emprego da simulação numérica além de proporcionar a garantia e segurança dos resultados, possibilita a realização de uma análise ágil e menos dispendiosa, sem a necessidade de envolvimento de mão de obra qualificada para preparo e execução das amostras e da análise experimental, como também sem a necessidade da aquisição de material para este fim, tendo somente as propriedades elásticas do compósito e suas resistências à ruptura fornecidas pela literatura, tornando assim tal processo menos trabalhoso, demorado e dispendioso. Portanto concluiAnais do 10o Congresso Brasileiro de Polímeros - Foz do Iguaçu, PR - Outubro/2009 se que a simulação numérica pode ser empregada para efeito de redução de custo e otimização no projeto de determinada estrutura.

De acordo com o exposto acima e considerando a complexidade de um laminado compósito, pode-se constatar a necessidade de conhecimento do comportamento mecânico que o projetista deve ter tanto em relação aos esforços que estão sendo aplicados no laminado em estudo como de sua reação a estes esforços, bem como de seu conhecimento quanto aos critérios de falhas mais adequados a serem empregados na análise do compósito, sem o qual seria inapropriado o emprego desta simulação com a finalidade de complementar/substituir em alguns casos, ou seja, quando já se tem conhecimento do comportamento mecânico do compósito em literaturas afins, para baratear a análise do laminado. 


\section{Agradecimentos}

Os autores agradecem a empresa holandesa Tencate pelo fornecimento dos laminados de PPS e à FAPESP pelo auxílio financeiro.

\section{Referências Bibliográficas}

1. BOtelho, E. C. Compósitos Aeronáuticos Processados a partir de Fibras de Carbono Impregnadas com Poliamida 6/6 via Processo de Polimerização Interfacial. Tese de Doutorado em Ciência no Curso de Engenharia Aeronáutica e Mecânica do Instituto Tecnológico de Aeronáutica (ITA), São José dos Campos/SP, 2002.

2. CHEONG Ng, Y. Deriving Lamina Properties from Laminate Properties using Classical Lamination Theory and Failure Criteria. Journal of Composite Materials Vol. 39 no. 1295, 2005.

3. CostA, G. G. Avaliação da Influência dos Ciclos Térmicos nas Propriedades dos Compósitos Termoplásticos de PPS e PEI com Fibras de Carbono e Vidro Conformados por Prensagem a Quente. Dissertação de Mestrado em Engenharia Aeronáutica e Mecânica, Instituto Tecnológico de Aeronáutica, São José dos Campos/SP, 2006.

4. TITA, V.; CARVAlHO, J.; SANTOS, N. C. Estudo do Comportamento Mecânico de Materiais Compósitos utilizando o Método dos Elementos Finitos. II congresso Nacional de Engenharia Mecânica. João Pessoa/PB, 2002.

5. DE FARIA, M. C. M. Avaliação do Efeito Higrotérmico nas Propriedades Mecânicas de Compósitos PPS/Fibras Contínuas. Dissertação de Mestrado em Engenharia Mecânica. UNESP - Universidade Paulista, Guaratinguetá/SP, 2008. Anais do 10o Congresso Brasileiro de Polímeros - Foz do Iguaçu, PR - Outubro/2009

6. DeTERESA, S. J.; LARSEN, G. J. Reduction in the Number of Independent Parameters for the TSAI-WU Tensor Polynomial Theory of Strength for Composite Materials. Journal of Composite Materials vol. 37 no. 1769, 2003.

Informações bibliográficas:

Conforme a NBR 6023:2002 da Associação Brasileira de Normas Técnicas (ABNT), este texto científico publicado em periódico eletrônico deve ser citado da seguinte forma: SIQUEIRA, Eder José; FARIA Maria Cândida Magalhães de; SILVA Fernando de Azevedo; BOTELHO Edson Cocchieri. Estudo dos critérios de falhas em flexão em Compósitos PPS/Fibras de Carbono utilizando elementos finitos. Cadernos UniFOA. Volta Redonda, ano IV, n. 11, dezembro 2009. Disponível em: <http://www.unifoa.edu.br/cadernos/edicao/11/11.pdf>

Endereço para Correspondência:

Eder Jose Siqueira

Curso de Engenharia Civil - UniFOA

ederana@ig.com.br

Centro Universitário de Volta Redonda

Campus Três Poços

Av. Paulo Erlei Alves Abrantes, $n^{\circ}$ 1325,

Três Poços - Volta Redonda / RJ

CEP: $27240-560$
Método dos Elementos Finitos. Revista Ciência e Engenharia vol. 10(2) p. 155-164, 2001.

8. HUYBRECHTS, S.; MAJI, A.; LAO, J.; WEGNER, P.; MEINK, T. Validation of the Quadratic Composite Failure Criteria with OutOf-Plane Shear Terms. Journal of Composite Materials. Vol. 36, no. 1879, 2002.

9.LABEAS,G.;BELESIS,S.;STAMATELOS, D. Interaction of Damage Failure and PostBuckling Behavior of Composite Plates with Cut-Outs by Progressive Damage Modelling. Available online www.sciencedirect.com. Elsevier ScienceDirect Composite Part B Vol. 10. LASPALAS, M.; CRESPO, C.; JIMÉNEZ, M. A.; GARCÍA, B.; PELEGAY, J. L. Application of Micromechanical Models for Elasticity and Failure to Short Fibre Reinforced Composites. Numerical Implementation and Experimental Validation. Composites and Structures Vol. 86 p. 977-987, 2008. 